\title{
An Research to Enhance the Old Manuscript Resolution using Deep Learning Mechanism
}

\author{
Syed Nawaz Pasha, D. Ramesh, D.Kodhandaraman, M D. Salauddin
}

\begin{abstract}
In this paper we address the problem of enhancing the resolution of old manuscripts as these will deteriorate over time. The manuscripts are of at most importance as they may contain sensitive and important imformation.Hence digital processing techniques play a crucial role to improve the quality of the manuscripts. We propose a standard deep learning mechanism that is conventional neural network (CNN) to enhance image resolution which will help to increase resolution of old manuscript .It not only increases the resolution but also tries to identify missing content using digit recognizer. We also compare the efficiency of our approach with the existing image enhancing techniques.
\end{abstract}

Keywords - deep learning, conventional neural network, Image enhancement, digit recognizer.

\section{INTRODUCTION}

Image enhancement is the process of adjusting digital images so that the results are more suitable for display or further image analysis. So we are using image enhancement technique to improve resolution of old manuscript. The old manuscripts are important documents which are hand written and which will deteriorate overtime. Moreover these old manuscripts their texture which makes it unable to identify what is written in them. Hence by using deep learning methodologies we try to improve the manuscript resolution and also try to uncover the text or digits in these manuscripts. This paper presents an approach to improve the old manuscript resolution by using opencv python libraries to read the manuscript as images and then apply CNN to increase the resolution.

\section{RELATED WORK}

Image enhancement techniques are wide spread in many applications of image processing where the quality of images is important for human interpretation.

There are some fundamental kinds of picture upgrade devices basically adjust the difference or splendor of a picture or control the grayscale or the red-green-blue shading of a picture. A few kinds of essential channels additionally permit changing a shading picture to high contrast, or to a sepia-tone picture, or including special visualizations.

There are three types of methods for this state-of-the$\operatorname{art}[1]$ :

Revised Manuscript Received on April 12, 2019.

Syed Nawaz Pasha, Computer Science \& Engineering, S R Engineering College, Warangal, Telangana, India (Email : syed_nawaz_pasha@srecwarangal.ac.in)

D. Ramesh, Computer Science \& Engineering, S R Engineering College, Warangal, Telangana, India

Dr. D.Kodhandaraman, Computer Science \& Engineering, S R Engineering College, Warangal, Telangana, India

MD. Salauddin, Computer Science \& Engineering, S R Engineering College, Warangal, Telangana, India
1. Self-similarity based

2. Dictionary learning based

3. Deep learning based

Self-similarity based methods work on pairing lowresolution and high resolution patches to improve the quality of low resolution image. Our method focuses on deep learning where we use convolutional neural network and show that the deep learning based methods overcome the shortcomings of state-of-the-art methods.

"The deeper the better" doesn't work here. Our technique uses 3 convolutional-layers but doesn't show much of improvement for 4 to 5 layer. Thus, harnessing the power of GPU using less no of layers as we have to consider training efficiency and storage.

\section{PROPOSED SYSTEM}

We propose a convolution neural network which is having 3 convolutional layers parallel to considering efficiency and storage. Traditionally, the image is up-scaled using bi-cubic interpolation. But it leads to loss of texture due to excessive smoothness which leads to unnatural texture. Instead, we use discrete-wavelet transform for up scaling and back-projection technique to enhance the edges. The up-sampled image is then iteratively back projected using back-projection filter based on Laplacian of Gaussian (LOG) this gives a more natural texture of the image in smooth areas. The method also removes spurious colours along the edges.

Assumption:

If (high-resolution patch representation is in image domain):

Reshape each representation to form the patch.

We expect that the filters act like an averaging filter Else:

We expect, W3 behaves like

I. projecting the coefficient onto the image domain

II. Then averaging

We perform pre-processing on a low-resolution image and then try to upscale it to the desired size using bi-cubic interpolation. Let us denote the up-scaled image as Y.

We consider up-scaled image $\mathrm{Y}$ as input image on which we wish to learn a mapping $F$ which conceptually consists of 3 operations:

1. Patch Extraction \& Representation

2. Non-Linear Mapping

3. Reconstruction

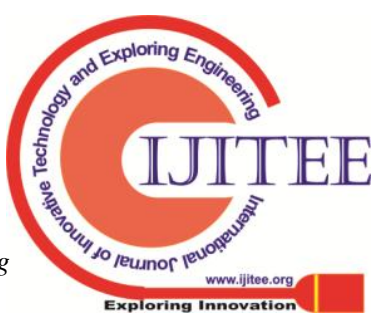




\subsection{Extraction of patches and image representation}

We extract patches from the image which is a strategy used for image restoration and apply below operation,

$\mathrm{f} 1(\mathrm{Y} 1)=\operatorname{maximum}(0, \mathrm{We} 1 * \mathrm{Y} 1+\mathrm{b} 1)$, and

We1 is $\mathrm{k} 1$ filters of $\mathrm{ch} \times \mathrm{m} 1 \mathrm{x} \mathrm{m} 2$ where ch number of channels in the image Y1

We1 applies k1 convolution.

$\mathrm{b} 1$ is $\mathrm{k} 1$ dimensional vectors associated with filters.

We have applied ReLU (maximum $(0$, , $x$ )) on the filter responses.

The ReLU belongs to the part of 2 nd operation.

\subsection{Non-Linear Mapping}

We are having $\mathrm{k} 1 \mathrm{dim}$ feature for each and every patch.we will map this $\mathrm{k} 1$-dim vectors to $\mathrm{k} 2$-dim one with filter size $1 \mathrm{x} 1$ by applying below operation,

$\mathrm{f} 2(\mathrm{Y} 1)=\max (0, \mathrm{We} 2 *(\mathrm{f} 1(\mathrm{Y} 1)+\mathrm{b} 2)$,

where We 2 contains $\mathrm{k} 2$ filters of size $\mathrm{k} 1 \mathrm{x} \mathrm{m} 2 \times \mathrm{m} 2$,

$\mathrm{b} 2$ is $\mathrm{k} 2$ dimensional.

\subsection{Reconstruct}

By applying below operation we construct the final image $\mathrm{f}(\mathrm{Y} 1)=\mathrm{We} 3 * \mathrm{f} 2(\mathrm{Y} 1)+\mathrm{b} 3$, where

We 3 contains ch filters of a size $\mathrm{k} 2 \times \mathrm{m} 3 \times \mathrm{m} 3$

b3 is ch-Dimensional Vector Learning:

To learn end to end mapping $f$ we need to find out the network parameters $\{\mathrm{We} 1, \mathrm{We} 2, \mathrm{We} 3, \mathrm{~b} 1, \mathrm{~b} 2, \mathrm{~b} 3\}$.

That can be accomplished by minimizing the loss between reconstructed image and respective ground truth image. We will take some set of images and train it.

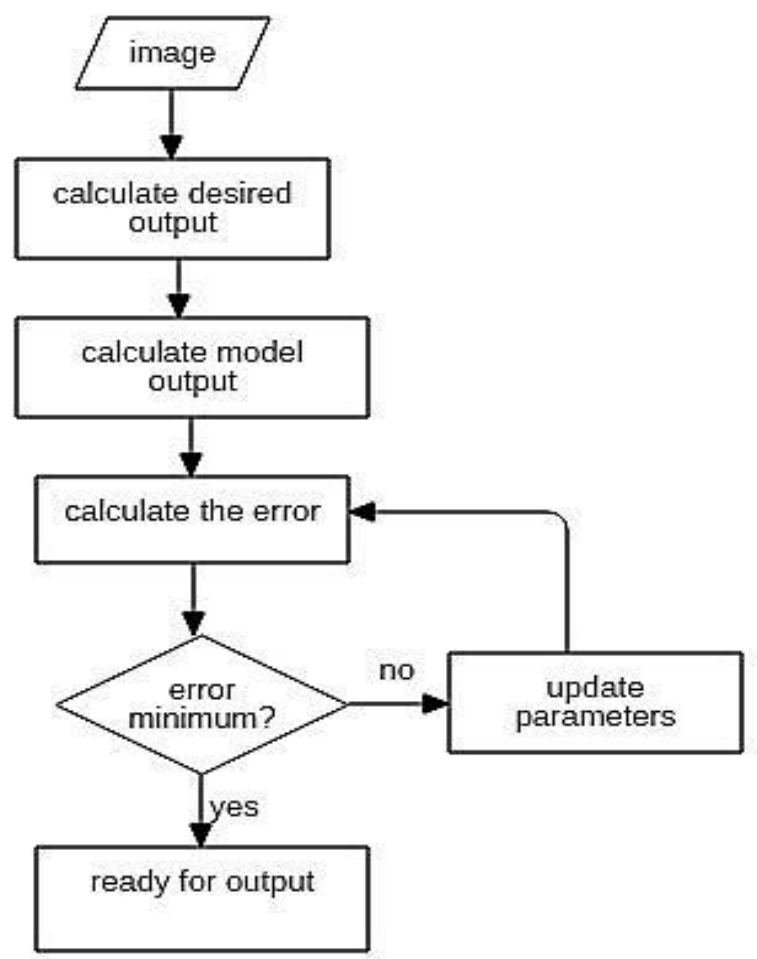

\subsection{Training model}

\section{DATA SETS TO BE USED FOR TESTING}
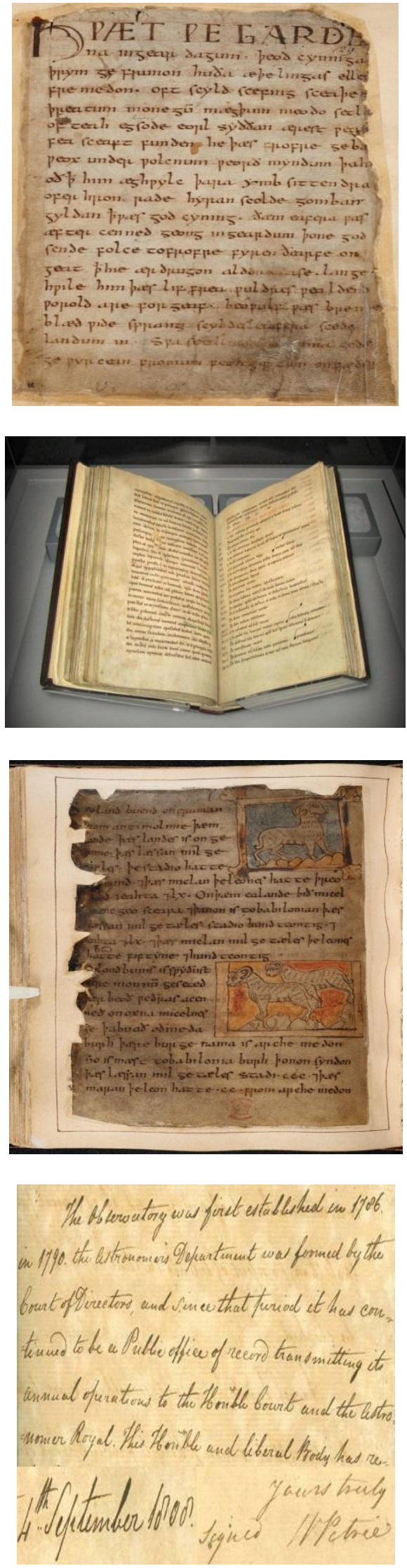


\section{TEST RESULT}

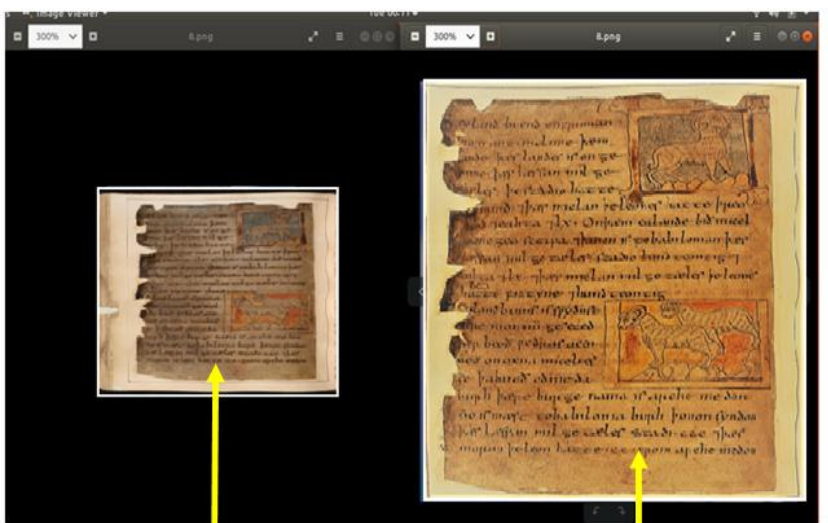

Input image

Output image

5. RESULT COMPARISON:

\begin{tabular}{|l|c|c|c|c|c|c|c|}
\hline Eval. Mat & Scale & Bicubic & NE+LLE [14] & KK [15] & ANR [16] & A+ [16] & CNN \\
\hline PSNR & 2 & 33.66 & 35.77 & 36.20 & 35.83 & 36.54 & 36.66 \\
\hline & 4 & 28.42 & 29.61 & 30.03 & 29.69 & 30.28 & 30.49 \\
\hline & & & & & & & \\
\hline Eval. Mat & Scale & Bicubic & NE+LLE [14] & KK [15] & ANR [16] & A+ [16] & CNN \\
\hline PSNR & 2 & 28.38 & 29.67 & 30.02 & 29.72 & 30.14 & 30.30 \\
\hline & 4 & 24.65 & 25.21 & 25.38 & 25.25 & 25.51 & 25.60 \\
\hline
\end{tabular}

5. CONCLUSION:

In this paper we compared few Images enhancement algorithms they offer a number of approaches for modifying images to achieve visually acceptable images. The decision of such strategies is a component of the particular errand, picture content, picture attributes, and review conditions. The results are captured and our approach improved the image resolution a little bit more than the other algorithms under study.

\section{REFERENCES}

1. K. I. Kim and Y. Kwon, "Single-image super-resolution using sparse regression and natural image prior," IEEE Trans. Pattern Anal. Mach. Intell., vol. 32, no. 6, pp. 1127-1133, Jun. 2010.

2. Image enhancement of old manuscripts using machine learning 2017 3rd International Conference on Applied and Theoretical Computing and Communication Technology (iCATccT)

3. Image Super-Resolution Using Deep Convolutional Networks, IEEE RANSACTIONS ON PATTERN ANALYSIS AND MACHINE INTELLIGENCE, VOL. 38, NO. 2, FEBRUARY 2016

4. K. Jia, X. Wang, and X. Tang, "Image transformation based on learning dictionaries across image spaces," IEEE Trans. Pattern Anal. Mach. Intell., vol. 35, no. 11, pp. 367-380, Feb. 2013.

5. Y. Jia, E. Shelhamer, J. Donahue, S. Karayev, J. Long, R Girshick, S. Guadarrama, and T. Darrell, "Caffe: Convolutional architecture for fast feature embedding," in Proc. ACM Int. Conf. Multimedia,2014, pp. 675-678.

6. Y. LeCun, L. Bottou, Y. Bengio, and P. Haffner, "Gradientbased learning applied to document recognition," Proc. IEEE, vol. 86, no. 11, pp. 2278-2324, Nov. 1998.

7. A. Krizhevsky, I. Sutskever, and G. Hinton, "ImageNet classification with deep convolutional neural networks," in Proc. Adv. Neural Inf. Process. Syst., 2012, pp. 1097-1105.

8. C. J. Schuler, H. C. Burger, S. Harmeling, and B. Scholkopf, "A machine learning approach for non-blind image deconvolution," in Proc. IEEE Conf. Comput. Vis. Pattern Recog., 2013, pp. 1067-1074. 